\title{
Tracheostomies for COVID-19 patients: a survey of 42 hospitals in Lombardy, Italy.
}

\author{
Davide Di Santo $^{1}$, alberto maria saibene ${ }^{2}$, Andrea Franzetti ${ }^{3}$, Giovanni Felisati ${ }^{4}$, and Mario \\ Bussi $^{1}$ \\ ${ }^{1}$ San Raffaele Hospital \\ ${ }^{2}$ University of Milan, Department of Health Sciences, Santi Paolo e Carlo Hospital \\ ${ }^{3}$ Ospedale Bassini \\ ${ }^{4}$ University of Milan
}

April 28, 2020

\section{KEYPOINTS}

- COVID-19 patients often require prolonged mechanical ventilation, and tracheostomy is a common choice.

- Shared guidelines for intensive care unit patient tracheostomies for COVID-19 patients do not exist.

- Our survey indicates the timing and technique of COVID-19 tracheostomies vary considerably among hospitals in Lombardy, Italy.

- Otolaryngologists are seldom involved with decision-making regarding tracheostomies for intensive care unit COVID-19 patients.

- Evidence-based interventions are essential for providing the best care to invasively ventilated COVID-19 patients.

\section{KEYWORDS}

SARS-Cov-2; COVID-19; coronavirus; tracheostomy; percutaneous tracheotomy; surgical tracheostomy; intubation; ventilation.

\section{INTRODUCTION}

After identifying the first Italian COVID-19 infected patient on 20 February 2020, a rapidly escalating infection cluster was discovered. On 21 February, a response coordinated by a governmental task force progressively led to a countrywide lockdown beginning on 9 March. Italy became the first Western country to address COVID-19, which on 20 March, the World Health Organization declared a pandemic.

Although pneumology, infectious disease, and intensive care units (ICUs), as well as emergency departments, have carried the heaviest healthcare burden during this outbreak, ${ }^{1}$ other departments must also address the increased infectious risk while meeting patient needs. Given the number of COVID-19 patients requiring long-term invasive ventilation, a surge in tracheostomies have ensued. Otolaryngologists have quickly become involved in patient management, despite previously having been 'second-line' specialists during infectious outbreaks. $^{2}$ This unprecedented need for tracheostomies reopened decades-old debates about ICU patient tracheostomy timing, techniques and operators: supporters of late versus early tracheostomies, percutaneous tracheostomies (PTs) versus open surgical tracheostomies (STs) and otolaryngologists versus anaesthesiologists. 
This unprecedented situation similarly affected all hospitals in the region, overburdening ICUs and inpatient units. Our study aimed to illustrate the COVID-19 healthcare situation and investigate ICU tracheostomy management decisions.

\section{MATERIALS AND METHODS}

We prepared a 13-item questionnaire asking the following: the number of COVID-19 patients treated, ICU dedicated beds, tracheostomies performed and their timing, preferred tracheostomy techniques with reasons for choosing PT or ST. The questionnaire was sent to each otolaryngology department in the Lombardy region, during the first week of April 2020. Department directors, instructed to collect data by collaborating directly with their respective ICUs, responded by phone the following week.

\section{Data collection and statistics}

Data were recorded on a Google Sheets spreadsheet (Google LLC, Mountain View, CA, US), anonymised and extracted. Descriptive statistics were performed by transferring data into an Excel 2010 spreadsheet (Microsoft Corp, Redmont, WA, US). Due to the small sample, data were considered as nonparametric.

\section{RESULTS}

The English translation of the full questionnaire with responses is available online as supporting information to this paper. Each director from the 42 Otolaryngology departments in Lombardy answered the questionnaire. Among them, two belonged to cancer centres and one to a paediatric hospital. All the hospitals, minus the two cancer centres, admitted COVID-19 patients into their wards and ICUs. The 40 hospitals accounted for almost 9000 hospital and approximately 1000 ICU COVID-19-dedicated beds. The two cancer centres were devoted to managing all complex cancer cases from the region, and patients were transferred whenever possible.

At the time of interview (six weeks after Italy's outbreak), over 500 tracheostomies were reported for intubated critical COVID-19 patients in Lombardy. In 38 of 40 involved hospitals, at least one procedure was performed. However, the number of performed procedures was inconsistent among centres, regardless of the number of long-term invasively ventilated patients. Notably, nearly half reported fewer than ten procedures.

In all cases, the anaesthesiologist determined a tracheostomy need, but we noticed tremendous time and preferred technique discrepancies among centres. We found the median time between symptom onset and ICU admission was $10 \pm 3,5$ days (range 1-18 days), and since then, the median time between intubation and tracheostomy was $12 \pm 4$ days (range 3-18 days). Pronation cycles were tried in each centre before prescribing tracheostomies but significantly differed among hospitals. A median of $3,5 \pm 1,5$ pronation cycles was performed before tracheostomy (range 1-18 cycles).

Concerning the preferred technique, 27 of 38 centres that reported at least one tracheostomy performed exclusively or mostly PTs. Among the remaining hospitals, in five cases PTs and STs were equally performed, whereas in six cases STs were preferred to PTs. No association was found between the number of performed procedures and preferred techniques.

Reasons for the chosen techniques are reported in Table 1. Operator preference was the primary determiner for choosing PTs. Additional reasons frequently cited were the superior postoperative management of the cannula and lower risk of complications. Open STs were mainly utilised for unfavourable anatomic conditions but also from a lack of experienced PT staff or PT kits.

\section{DISCUSSION}

Synopsis of key and new findings

The entire healthcare system helped manage the region's COVID-19 pandemic. Many patients required ICU stays, often with prolonged mechanical ventilation, resulting in nearly all centres to perform at least one tracheostomy. Notably, Lombardy's ICUs reached maximum occupancy, which required converting operating theatres into ICUs for invasive ventilation. ${ }^{1}$ This unprecedented situation forced the entire region to face the 
wide-scale clinical emergency without patient-treatment guidelines. We observed that non-existent criteria for performing tracheostomies on intubated COVID-19 patients result in significant discrepancies between their timing and techniques.

This survey revealed two distinct timing approaches: most centres reverted to tracheostomy for prolonged intubations, lasting more than 10-12 days, whereas some others performed tracheostomies after 5-6 days to accelerate weaning from mechanical ventilation. The latter group is among those performing a higher number of procedures.

Median reported ICU stay is nine days, ${ }^{3}$ therefore, waiting for respiratory disorder recovery seems reasonable. However, tracheostomy within seven days reduces the duration of mechanical ventilation and ICU stays as well as hospital-acquired pneumonia and mortality. ${ }^{4}$ Early tracheostomy also reduces the need for sedation and may accelerate rehabilitation. ${ }^{5}$ Further studies are needed to provide data for determining appropriate approach.

Percutaneous tracheostomy is the preferred technique, most often performed by anaesthesiologists, whereas otolaryngologists are required for STs in case of predicted anatomical difficulties. However, a closer collaboration between these specialists is advisable since each of the two techniques has its advantages. Percutaneous tracheotomies reduce the risk for major bleeding and stoma infection. This proves vital due to the high prevalence of multi-resistant bacteria in ICUs and the use of anticoagulants for COVID-19 patient because of suspected microvascular pulmonary thrombosis. ${ }^{6}$ Concurrently, recently proposed COVID-19-specific ST techniques reduce hypoxia time and minimise aerosol generation and operator infection. ${ }^{7-10}$ Furthermore, in an emergency setting, shortages of resources, PT-experienced staff and PT kits must be considered.

\section{Strengths and clinical applicability of the study}

This study was conducted in a region significantly affected by the COVID-19 pandemic, and data collected from each otorhinolaryngology departments actively addressing the situation. Presently, English literature neither provides research regarding COVID-19-related tracheostomies nor multicentre data. Therefore, our questionnaire offers a unique perspective, which emphasises the need for additional data to establish dedicated guidelines addressing multiple discrepancies that accompanied COVID-19 tracheostomies during the pandemic's early phases.

\section{CONCLUSION}

Treating ICU patient tracheostomies, especially during fragile situations such as COVID-19 infections, requires careful planning. Additionally, the present approaches need refinement and shared guidance. Although we cannot deny the pandemic's ramifications on the economy and human lives, the situation presents an opportunity to improve standards of care. The similarities among ICU patients admitted to various hospitals could help develop shared, evidence-based interventions for tracheostomies in long-term invasively ventilated COVID-19 patients. Consequently, the present lack of guidelines demands mandatory collaboration between otolaryngologists and anaesthesiologists to determine the timing and technical approaches appropriate for each patient.

\section{REFERENCES}

1. Grasselli G, Pesenti A, Cecconi M. Critical Care Utilization for the COVID-19 Outbreak in Lombardy, Italy: Early Experience and Forecast During an Emergency Response. JAMA 2020. doi:10.1001/jama.2020.4031

2. Saibene AM, Allevi F, Biglioli F, Felisati G. Role and Management of a Head and Neck Department during the COVID-19 Outbreak in Lombardy. Otolaryngol Head Neck Surg 2020 Apr 7 doi:10.1177/0194599820917914

3. Grasselli G, Zangrillo A, Zanella A, et al. Baseline Characteristics and Outcomes of 1591 Patients Infected With SARS-CoV-2 Admitted to ICUs of the Lombardy Region, Italy. JAMA 2020; Apr 6;e205394. doi:10.1001/jama.2020.5394

4. Adly A, Youssef TA, El-Begermy MM, Younis HM. Timing of tracheostomy in patients with prolonged endotracheal intubation: a systematic review. Eur Arch Otorhinolaryngol 2018;275:679-690. doi:10.1007/s00405-017-4838-7 
5. Brugliera L, Spina A, Castellazzi P, Cimino P, Tettamanti A, Houdayer E, Arcuri P, Alemanno F, Mortini P, Iannaccone S. Rehabilitation of COVID-19 patients. J Rehabil Med 2020; Apr 15;52(4):jrm00046. doi:10.2340/16501977-2678

6. Ciceri F, Beretta L, Scandroglio AM, et al. Microvascular COVID-19 lung vessels obstructive thromboinflammatory syndrome (MicroCLOTS): an atypical acute respiratory distress syndrome working hypothesis. Crit Care Resusc 2020; Apr 15.

7. Pichi B, Mazzola F, Bonsembiante A, Petruzzi G, Zocchi J, Moretto S, De Virgilio A, Pellini R. CORONA-steps for tracheotomy in COVID-19 patients: A staff-safe method for airway management. Oral Oncol. 2020; Apr 6;105:104682. doi:10.1016/j.oraloncology.2020.104682.

8. Jacob T, Walker A, Mantelakis A, Gibbins N, Keane O. A framework for open tracheostomy in COVID19 patients. Clin Otolaryngol 2020; Apr 13. doi:10.1111/coa.13549.

9. Canadian Society of Otolaryngology - Head and Neck Surgery. Recommendations from the CSO-HNS Taskforce on Performance of Tracheotomy During the COVID-19 Pandemic; 2020. https://www.entcanada.org/wpcontent/uploads/COVID-19-Guidelines-CSOHNS-Task-Force-Mar-23-2020.pdf. Accessed April 18, 2020.

10. Societa Italiana di Otorinolaringoiatria e Chirurgia Cervico-Facciale (SIO). Tracheostomia in Pazienti Affetti Da COVID-19; 2020. https://www.sioechcf.it/wp-content/uploads/2020/03/Riassunto-lineeguida-tracheo.pdf. Accessed April 18, 2020.

\section{Hosted file}

Table 1.docx available at https://authorea.com/users/314265/articles/444595-tracheostomies-forcovid-19-patients-a-survey-of-42-hospitals-in-lombardy-italy 